\title{
Industry relationships between physicians and professional medical associations: corrupt or essential?
}

\author{
C. Daniel Smith · Bruce V. MacFadyen
}

Published online: 20 January 2010

(C) Springer Science+Business Media, LLC 2010

\begin{abstract}
Congress and others have called into question the propriety of relationships between professional medical associations (PMAs) and industry. These relationships are critical to the continued development of new and better devices and procedures for patients. Better guidelines are needed to help guide these relationships. Overrestrictive regulatory oversight risks overconstraint of these relationships and hindrance to medical progress.
\end{abstract}

Keywords Education - Healthcare Policy · Conflcit of Interest · Professional Medical Association

Recently, the propriety of relationships between industry and professional medical associations has been called into question [1, 2]. In particular, Washington politicians and editors of leading medical journals have advocated for regulation of these relationships, urging that they be severely truncated, and in many cases eliminated entirely.

Although corrupt relationships between physicians and industry truly can be shown by examples, especially situations in which potential conflicts of interest were not revealed or recognized, these cases alone should not be used as the basis for excessive regulatory infringement. The involvement of physicians and professional medical associations with the development and deployment of new drugs and devices has been imperative to the progress

C. D. Smith $(\bowtie)$

SAGES, Mayo Clinic in Florida, 4500 San Pablo Road,

Jacksonville, FL 33224, USA

e-mail: smith.c.daniel@mayo.edu

B. V. MacFadyen

SAGES, Department of Surgery, Medical College of Georgia,

BI 4076, Augusta, GA 30912, USA realized by the medical profession in providing better care to patients. This is especially true for surgeons and medical devices.

Several important points must be highlighted in considering the issue of regulating the relationships between industry and individual physicians or professional medical associations (PMAs). These points include recognizing the differences between the medical device industry and the pharmaceutical industry, the inseparable relationship of innovation and device development with the device industry, the delineation of research and development (R\&D) and education from sales and marketing, and the critical necessity of disclosure and transparency. The following list summarizes these distinctions:

- The medical device industry and the pharmaceutical industry are vastly different.

- Physician innovation and the device development industry are inseparable.

- $R \& D$ and education must be differentiated from industry sales and marketing.

- Disclosure and transparency are critical.

In considering the involvement of medical professionals in the development of new treatments, it is critical to remember that device development is vastly different from pharmaceutical development. Pharmaceutical products often are developed by researchers employed directly by the company or through an academic or commercial laboratory, a process largely devoid of the practicing physician's direct involvement. The pharmaceutical company expends considerable in-house resources before a drug is available for potential clinical use. Only after the drug is fully developed does the practicing physician use it in pharmaceutical industry-directed drug trials to establish safety and efficacy. Often, the investigator or the 
investigator's institution is compensated in return for his or her participation in such trials, including the expectation of publishing favorable results. At this point in the life of a new drug, the pharmaceutical company has considerable incentive to see the drug reach the market so it can recover its significant up-front investment. In this setting, the physician's role is more focused on confirmation of the company's claims and publication of satisfactory results, and not so much on the development or refinement of the drug.

In contrast, a device company rarely develops a device with in-house resources, but rather, relies on individual physicians to bring forward concepts for devices and then participate actively with in-house engineers in both developing and improving the product. In this, device companies do not maintain a significant in-house staff of scientists and researchers. Instead, they employ engineers who can translate the ideas and needs of practicing physicians into device prototypes. Thus, the device company's resources are more heavily leveraged to identify and explore clinical needs as defined by procedure-oriented physicians such as surgeons or endoscopists and to refine device prototypes to meet clinical needs. It is this direct relationship with the physician that allows the development and advancement of medical devices. Device companies and their engineers cannot independently develop devices for medical care. This device industry-physician relationship has been essential to the advancement of procedural medicine in recent decades, and without it, we will see a significant decline in the number and quality of new devices developed.

Another critical element in the PMA and industry relation discussion is clearly differentiating the process of device R\&D and education from the company's sales and marketing. As outlined earlier, device companies cannot develop new devices without close relationships with practicing physicians. Once a new device is developed and ready for use, considerable education and training often is needed to introduce it safely. The physician's role in education and training is critical, and without direct physician involvement in the designing of education and training programs, a new device may cause significant harm when introduced into practice. Professional medical associations have a particularly important role to play in the process of educating and training physicians in the use of new devices. Continued relationships between PMAs and industry are essential to the continued safe deployment of new medical devices and techniques to the practicing physician.

In contrast, the role of the physician and the PMA in sales and marketing of the device should be carefully monitored and managed. A clear and publically visible line should be drawn to separate device $R \& D$ and education from sales and marketing. It is in this area that much of the inappropriate behavior surrounding device development has been identified. Surgical techniques have significantly changed in the past 20 years and will continue to develop rapidly in the foreseeable future. Rapid changes in new technology increase the potential for financial abuse, often due to the clinician's enthusiasm for the new technology and the potential to be on the "cutting edge."

In a recent publication, Chatterji et al. [3] indicate that product development requires discovery, development, and dissemination. They note that authors citing a particular device in medical journals have a great impact on the marketing and adoption of the device. It is estimated that one citation can increase the product's market value by $3 \%$, thus emphasizing the importance of ethical and fair guidelines in the industry-physician relationship, with all parties complying.

In a widely publicized example, researchers at Cleveland Clinic published their experience with a new device to treat atrial fibrillation. The results with this device were remarkable, and the company quickly saw its stock price rise. Unfortunately, the researchers had significant financial interests in the device and the company. This was not discovered until after the publication, calling into question the credibility of the research and the device itself. Avoiding the comingling of sales and marketing interests with R\&D and education will help to prevent confusion or the inappropriate influence of industry over physician decisions or behavior.

All this speaks to the issue of disclosure and transparency. Disclosure of any and all relationships between individual physicians or PMAs and industry must be fully declared and transparent. As noted earlier, anything less is to conceal a potential influence that might bias the opinions or decisions of clinicians, especially when considering the presentation or publishing of their experience with devices or when making recommendations or providing educational material. Complete disclosure of any industry relationship is essential to avoiding any conflict of interest, real or perceived.

Unfortunately, medical schools and other leading health care organizations have not done an adequate job of identifying and managing conflict of interest. In 2008, Ehringhaus et al. [4], surveyed 125 medical schools in the United States regarding their conflict of interest policies. Of the 86 medical schools $(69 \%)$ responding, 38\% reported that they had a written conflict of interest policy, whereas $37 \%$ were developing one, and $25 \%$ had not developed any guidelines.

These data indicate that even educational institutions, often those receiving government grants under the highest regulation and scrutiny, have been lax in establishing and enforcing appropriate conflict of interest guidelines. This 
needs to change, and PMAs in particular should take steps to ensure public disclosure and transparency for all of their proceedings, publications, and educational offerings. Anything less leaves us all at risk for confounded data and decisions about how we care for patients.

In conclusion, the relationships between physicians or PMAs and industry have been essential to the advancement of medical science. Notwithstanding, examples exist in which these relationships have led to behaviors among both physicians and industry that were conflicted by personal and financial gains. Without question, greater scrutiny and transparency are necessary to ensure appropriate ongoing relationships between physicians or PMAs, industry, and the patient. However, severe regulation and limitation of these interactions risks slowing or even halting medical progress, particularly that of devices. All PMAs, medical institutions, and medical journals must establish and enforce conflict of interest guidelines that focus on differentiation of R\&D and education from sales and marketing, disclosure and transparency in all industry relationships, and compliance by all their members.

In addition, PMAs must require all officers and committee members to identify clearly any industry relationships and potential conflicts of interest. Publications in peer-reviewed journals must include a complete list of potential conflicts of interest from the authors. The course literature of continuing medical education must clearly identify speakers and their potential conflicts of interest in any content presented. By embracing these concerns, real or perceived, and taking steps to manage conflict of interest, we can ensure preservation of these valuable relationships and avoid regulatory overinfringement.

Disclosures C. Daniel Smith and Bruce MacFadyen have no conflicts of interest or financial ties to disclose.

\section{References}

1. Rothman DJ, McDonald WJ, Berkowitz CD, Chimonas SC, DeAngelis CD, Hale RW, Nissen SE, Osborn JE, Scully JH Jr, Thomson GE, Wofsy D (2009) Professional medical associations and their relationships with industry: a proposal for controlling conflict of interest. JAMA 301:1367-1372

2. Institute of Medicine (IOM) (U.S.) (2009) Conflict of interest in medical research education and practice. National Academy of Sciences, Washington DC

3. Chatterji AK, Fabrizio KR, Mitchell W, Schulman KA (2008) Physician-industry cooperation in the medical device industry. Health Aff 27:1532-1543

4. Ehringhaus SH, Weissman JS, Sears JL, Goold SD, Feibelmann S, Campbell EG (2008) Responses of medical schools to institutional conflicts of interest. JAMA 299:665-671 